\title{
One-loop radiative corrections to the QED Casimir energy
}

\author{
Reza Moazzemia ${ }^{\mathrm{a}}$, Amirhosein Mojavezi \\ Department of Physics, University of Qom, Ghadir Blvd., Qom 371614-611, Islamic Republic of Iran
}

Received: 2 February 2016 / Accepted: 20 April 2016 / Published online: 18 May 2016

(C) The Author(s) 2016. This article is published with open access at Springerlink.com

\begin{abstract}
In this paper, we investigate one-loop radiative corrections to the Casimir energy in the presence of two perfectly conducting parallel plates for QED theory within the renormalized perturbation theory. In fact, there are three contributions for radiative corrections to the Casimir energy, up to order $\alpha$. Only the two-loop diagram, which is of order $\alpha$, has been computed by Bordag et. al (Ann. Phys. 165:192, 1985), approximately. Here, up to this order, we consider corrections due to two one-loop terms, i.e., photonic and fermionic loop corrections resulting from renormalized QED Lagrangian, more precisely. Our results show that only the fermionic loop has a very minor correction and the correction of photonic loop vanishes.
\end{abstract}

\section{Introduction}

The Casimir effect is a physical manifestation of changes in the zero point energy of a quantum field for different configurations. The zero point configuration refers to one in which there does not exist any on-shell physical excitation of the field.

In 1948 Casimir predicted the existence of this effect as an attractive force between two infinite parallel uncharged perfectly conducting plates in vacuum [1]. This effect was observed experimentally by Sparnaay [2] and Arnold et al [3] (for a general review on the Casimir effect, see Refs. [4,5]). Similar measurements have been done for other geometries, and their precisions have been greatly improved [6-11]. The manifestations of the Casimir effect have been studied in many different areas of physics. For example, the magnitude of the cosmological constant has been estimated using the Casimir effect [12-14]. This effect has been also studied within the context of string theory [15]. It has been investigated in connection with the properties of the spacetime with extra dimensions [16-18]. The majority of the investi-

\footnotetext{
a e-mail: r.moazzemi@qom.ac.ir
}

gations related to the Casimir effect concerns with the calculation of this energy or the consequence forces for different fields in different geometries, such as parallel plates [1,19], cubes [20-28], cylinders [27,29-31], and spherical geometries [27,32-34].

Although the Casimir effect has been known for nearly 70 years, the question of what are the leading radiative corrections to this effect is still a subject of discussion. The first endeavors to compute the radiative corrections to the Casimir energy were reported in a paper by Bordag, Robaschik, and Wieczorek (BRW) [35]. There exist many works on the radiative corrections to the Casimir energy for various cases (see for example [35-48]). In the case of a real massive scalar field, Next to Leading Order (NLO) correction to the energy has been computed in [4,28,44-53]. Moreover, the twoloop radiative corrections for some effective field theories have been investigated in [40-42]. Bordag and his collaborators have approximately calculated radiative correction to the Casimir energy due to one of the three related terms of order of $\alpha$, in the presence of two perfectly conducting parallel plates for QED theory. In this viewpoint, the photon propagator satisfies boundary conditions on the plates, while the plates are transparent to the electrons. They found the correction $E^{(1)}=\frac{\pi^{2} \alpha}{2560 m a^{4}}$ to the popular leading term of Casimir energy (per unit area) $E_{\mathrm{em}}^{(0)}=-\frac{\pi^{2}}{720 a^{3}}$, where $a$ is the distance between plates and $m$ is the electron mass. In 1998 this result with another approach has been reported [36-39]. Although they postulate no boundary conditions for the electron field because such conditions would lead to additional contributions in zeroth order which have not been observed, the fermionic term [54], $E_{\text {fermion }}^{(0)}=-\frac{m^{2}}{4 \pi^{2} a} \sum_{n=1}-\frac{1}{j^{2}}\left[2 K_{2}(2 a m j)-K_{2}(4 a m j)\right]$ is exist (Here, $K_{n}(x)$ is the modified Bessel function of order $n)$. However, due to its Yukawa form for the large mass case, $E_{\text {fermion }}^{(0)} \sim-\frac{m^{2}}{4 \pi^{2} a} \sqrt{\frac{\pi}{m a}} e^{-2 m a}$, at distances much larger than a few Compton wave length of electron it has really too small value to be observed. 
In the context of perturbation theory we need the renormalization to compute loop diagrams. There are two completely equivalent methods for renormalization; first, bare perturbation theory: working with the bare parameters and relate them to their physical values at the end of calculations, second, renormalized perturbation theory: using counterterms at the starting point to absorb unphysical part of parameters. Both of them need renormalization conditions to fix the infinities in certain conditions. The differences between two renormalization procedures are purely a matter of bookkeeping. In the framework of renormalized perturbation theory for QED there are three vacuum bubbles of order of $\alpha$. Up to now, all the papers on the Casimir effect, that we are aware of, have not been calculated two of those, namely: photonic loop resulting from electromagnetic field and fermionic loop $\otimes$ related to the spinor field. Note that, although according to the common understanding we use the same counterterms for two different situations (with and without plates), the difference of vacuum energies may still be nonzero due to the difference of boundary conditions applying on loop propagators.

The main purpose of this paper is to directly calculate radiative correction to the Casimir energy resulting from one-loop corrections namely: one-loop photon and one-loop fermion, in the framework of the renormalized perturbation theory for QED theory. These corrections are of order $\alpha$. In order to do this, we use Green's functions in the presence of plates for Electromagnetic field with Dirichlet boundary condition and for spinor field with MIT bag boundary condition as propagators. Our main regularization is dimensional regularization.

Our approach for the calculation of radiative corrections to Casimir energy is the most direct one. In this way we subtract two infinite energies: one relate to presence and the other without presence of two plates. We adjust both of their regulators in such a way that the divergences removed and the physical result is obtained.

To have a throughout complete correction, up to order $\alpha$, one must also compute two-loop term once the fermionic field is submitted to MIT bag boundary conditions. However, it is notable that almost all the Casimir forces for various massive fields, which precisely calculated in the literature, have Yukawa asymptotic forms (usually $K_{n}(m a)$ ) even for leading term in different dimensions. Therefore, here we only calculate the one-loop diagram which seems more important than two-loop one that has two fermion propagators.

We organized the paper as follows: In Sect. 2 we briefly review the renormalization of quantum electrodynamics. In Sect. 3 using analogies between an electromagnetic field and a massless scalar field, photonic loop correction is considered. We use the Dirichlet boundary condition on the two plates. In Sect. 4 we directly calculate radiative correction to the Casimir energy resulting from fermionic loop where MIT bag boundary condition, as constraints on both of the plates, is considered. In Sect. 5 we summarize our results and state our conclusions.

\section{Renormalization of quantum electrodynamics: a brief review}

In this section we briefly review systematics of renormalization for QED theory (see for complete details [55]). The original QED Lagrangian is

$\mathcal{L}_{\mathrm{QED}}=-\frac{1}{4}\left(F_{\mu \nu}\right)^{2}+\bar{\psi}\left(i \not \partial-m_{0}\right) \psi-e_{0} \bar{\psi} \gamma_{\mu} \psi A^{\mu}$

By replacing $\psi=z_{2}^{\frac{1}{2}} \psi_{r}$ and $A^{\mu}=z_{3}^{\frac{1}{2}} A_{r}^{\mu}$, it becomes

$$
\begin{aligned}
\mathcal{L}_{\mathrm{QED}}= & -\frac{1}{4} z_{3}\left(F_{\mu \nu}^{r}\right)^{2}+z_{2} \bar{\psi}_{r}\left(i \not \partial-m_{0}\right) \psi_{r} \\
& -e_{0} z_{2} z_{3}^{\frac{1}{2}} \bar{\psi}_{r} \gamma_{\mu} \psi_{r} A_{r}^{\mu},
\end{aligned}
$$

where $e_{0}$ is the bare electric charge and $z_{2}$ and $z_{3}$ are the field-strength renormalizations for $\psi$ and $A^{\mu}$ respectively. We define a scaling factor $z_{1}$ as follows:

$e z_{1}=e_{0} z_{2} z_{3}^{\frac{1}{2}}$.

We can split each term of the Lagrangian into two pieces as follows:

$$
\begin{gathered}
\mathcal{L}_{\mathrm{QED}}=-\frac{1}{4}\left(F_{\mu \nu}^{r}\right)^{2}+\bar{\psi}_{r}(i \not \partial-m) \psi_{r}-e \bar{\psi}_{r} \gamma^{\mu} \psi_{r} A_{r}^{\mu} \\
-\frac{1}{4} \delta_{3}\left(F_{r}^{\mu \nu}\right)^{2}+\bar{\psi}_{r}\left(i \delta_{2} \not \partial-\delta_{m}\right) \psi_{r}-e \delta_{1} \bar{\psi} \gamma_{\mu} \psi_{r} A_{r}^{\mu},
\end{gathered}
$$

where $\delta_{3}=z_{3}-1, \delta_{2}=z_{2}-1, \delta_{m}=z_{2} m_{0}-m$ and $\delta_{1}=z_{1}-1=\left(\frac{e_{0}}{e}\right) z_{2} z_{3}^{\frac{1}{2}}-1$ are counterterms. Here, $m$ and $e$ are the physical mass and physical charge of the electron which measured at large distances. Now, the Feynman rules for this Lagrangian are
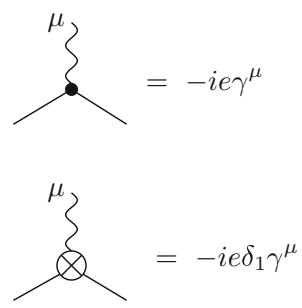

$$
\begin{aligned}
& \sim \widetilde{\vec{k}} \sim \nu=\frac{-i}{k^{2}+i \epsilon}\left(g^{\mu \nu}-(1-\xi) \frac{k^{\mu} k^{\nu}}{k^{2}}\right) \\
& \mu \sim \otimes \sim \nu=-i\left(g^{\mu \nu} k^{2}-k^{\mu} k^{\nu}\right) \delta_{3}
\end{aligned}
$$




$$
\begin{gathered}
\vec{p}=\frac{i}{\not \supset-m+i \epsilon} \\
\longrightarrow \otimes \rightarrow \quad=i\left(\not p \delta_{2}-\delta_{m}\right) .
\end{gathered}
$$

Each of the four counterterms must be fixed by renormalization conditions. For QED theory these conditions are (see please [55])

$$
=-i \Sigma(p)
$$

In the above equations $-i \Sigma(p)$ denotes the sum of all oneparticle irreducible (1PI) diagrams with two external fermion lines. By pretending that the photon has a small nonzero mass $\mu$ to control the infrared divergences, up to leading order in $\alpha$, the one-loop diagram contributing to $-i \Sigma(p)$ becomes

$$
\begin{aligned}
& -i \Sigma(p) \underset{\mathcal{O}(\alpha)}{=}-e^{2} \int_{0}^{1} \mathrm{~d} x \\
& \times \int \frac{d^{4} l}{(2 \pi)^{4}} \frac{-2 x \not p+4 m}{\left[l^{2}-x(1-x) p^{2}-x \mu^{2}-(1-x) m^{2}\right]^{2}} .
\end{aligned}
$$

One can evaluate the diagrams in dimensional regularization. If fact, we compute them as an analytic function of the dimensionality of spacetime $d$. The final expression for any observable quantity should have a well-defined limit as $d \rightarrow 4$. Up to leading order in $\alpha, i \Sigma(p)$ becomes

$$
\begin{aligned}
& -i \Sigma(p)=-i \frac{e^{2} m}{(4 \pi)^{\frac{d}{2}}} \int_{0}^{1} \mathrm{~d} x \\
& \times \frac{\Gamma\left(2-\frac{d}{2}\right)}{\left((1-x)^{2} m^{2}+x \mu^{2}-x(1-x) p^{2}\right)^{2-\frac{d}{2}}} \\
& \quad \times[(4-\epsilon) m-(2-\epsilon) x p] .
\end{aligned}
$$

with $\epsilon=4-d$. Since we prefer to work with dimensionless parameters we convert this formula as

$$
\begin{aligned}
& -i \Sigma(p)=-i \frac{e^{2}}{a^{d-3}(4 \pi)^{\frac{d}{2}}} \int_{0}^{1} \mathrm{~d} x \\
& \times \frac{\Gamma\left(2-\frac{d}{2}\right)}{\left((1-x)^{2} \tilde{m}^{2}+x \tilde{\mu}^{2}-x(1-x) \tilde{p}^{2}\right)^{2-\frac{d}{2}}} \\
& \times[(4-\epsilon) \tilde{m}-(2-\epsilon) x \tilde{p}],
\end{aligned}
$$

where $\tilde{l}=l a, \tilde{p}=p a, \tilde{\mu}=\mu a, \tilde{m}=m a$. Here $1 / a$ is an arbitrary scale with mass dimension 1 (in the problem of Casimir effect $a$ can be the plates separation.)

Moreover, $i \Pi\left(k^{2}\right)$ defines the sum of all 1PI insertions into the photon propagator and up to order $\alpha$ becomes

$$
\begin{aligned}
\Pi\left(k^{2}\right)= & \frac{-e^{2}}{a^{d-4}(4 \pi)^{\frac{d}{2}}} \int_{0}^{1} \mathrm{~d} x \\
& \times \frac{\Gamma\left(2-\frac{d}{2}\right)}{\left(\tilde{m}^{2}-x(1-x) \tilde{k}^{2}\right)^{2-\frac{d}{2}}} 8 x(1-x),
\end{aligned}
$$

where $\tilde{k}=k a$. In Eq.(13), $\Gamma^{\mu}\left(p^{\prime}, p\right)$ denotes the sum of vertex diagrams. More accurately

$\Gamma^{\mu}\left(p^{\prime}, p\right)=\gamma^{\mu} F_{1}\left(k^{2}\right)+\frac{i \sigma^{\mu \nu} k_{v}}{2 m} F_{2}\left(k^{2}\right)$,

where $F_{1}$ and $F_{2}$ are unknown functions of $k^{2}$ called form factors and $\sigma^{\mu \nu}=\frac{i}{2}\left[\gamma^{\mu}, \gamma^{\nu}\right]$. To lowest order, $F_{1}=1$ and $F_{2}=0$, we have $\Gamma^{\mu}=\gamma^{\mu}$. By using Eqs. (16), (17) and (18), up to leading order in $\alpha$, the counterterms are derived as follows:

$$
\begin{aligned}
\delta_{3}= & \frac{-e^{2}}{a^{d-4}(4 \pi)^{\frac{d}{2}}} \int_{0}^{1} \mathrm{~d} x \frac{\Gamma\left(2-\frac{d}{2}\right)}{\left(\tilde{m}^{2}\right)^{2-\frac{d}{2}}} 8 x(1-x), \\
\delta_{m}= & \frac{\tilde{m} \delta_{2}}{a^{d-3}}-\frac{e^{2} \tilde{m}}{a^{d-3}(4 \pi)^{\frac{d}{2}}} \int_{0}^{1} \mathrm{~d} x \frac{\Gamma\left(2-\frac{d}{2}\right)}{} \\
& \quad \times\left[(1-x)^{2} \tilde{m}^{2}+x \tilde{\mu}^{2}\right]^{2-\frac{d}{2}}(4-2 x-\epsilon(1-x)),
\end{aligned}
$$

$$
\begin{aligned}
\delta_{2} & =\frac{-e^{2}}{a^{d-4}(4 \pi)^{\frac{d}{2}}} \int_{0}^{1} \mathrm{~d} x \frac{\Gamma\left(2-\frac{d}{2}\right)}{\left[(1-x)^{2} \tilde{m}^{2}+x \tilde{\mu}^{2}\right]^{2-\frac{d}{2}}} \\
& \times\left[(2-\epsilon) x-\frac{\epsilon}{2} \frac{2 x(1-x) \tilde{m}^{2}}{(1-x)^{2} \tilde{m}^{2}+x \tilde{\mu}^{2}}(4-2 x-\epsilon(1-x))\right],
\end{aligned}
$$

$$
\begin{aligned}
\delta_{1}= & \frac{-e^{2}}{a^{d-4}(4 \pi)^{\frac{d}{2}}} \int_{0}^{1} \mathrm{~d} z(1-z)\left\{\frac{\Gamma\left(2-\frac{d}{2}\right)}{\left((1-z)^{2} \tilde{m}^{2}+z \tilde{\mu}^{2}\right)^{2-\frac{d}{2}}}\right. \\
& \times \frac{(2-\epsilon)^{2}}{2}+\frac{\Gamma\left(3-\frac{d}{2}\right)}{\left[(1-z)^{2} \tilde{m}^{2}+z \tilde{\mu}^{2}\right]^{3-\frac{d}{2}}} \\
& \left.\times\left[2\left(1-4 z+z^{2}\right)-\epsilon(1-z)^{2}\right] \tilde{m}^{2}\right\} .
\end{aligned}
$$

According to the above discussion three vacuum bubbles contribute to the Casimir energy: \&3, \&. Two first diagrams arise from Eqs. (8) and (10). Bordag et al. have computed only the last one, though approximately. In the next two sections we will consider the effect of the other two vacuum bubbles. 


\section{Photonic loop}

In this section, we calculate NLO radiative correction to the Casimir energy due to the photonic loop. We use Dirichlet boundary condition on the two parallel perfectly conducting plates in $(3+1)$ dimensions. Although electromagnetic field cannot be submitted to Dirichlet boundary conditions itself, one can describe the TE and TM modes of the electromagnetic field in the presence of the conducting plates as two scalar fields submitted to Dirichlet boundary conditions. Obviously in the presence of the two plates, propagators automatically incorporate the boundary conditions and are position dependent. The contribution of one-loop photon to the vacuum energy in the interval $\left(\frac{-a}{2}, \frac{a}{2}\right)$ is

$\Delta E_{\mathrm{Ph}}=\int_{-a / 2}^{a / 2} d^{3} \mathbf{x}\left\langle\Omega\left|\mathcal{H}_{I}\right| \Omega\right\rangle=i \int_{-a / 2}^{a / 2} d^{3} \mathbf{x}+\mathcal{O}\left(\alpha^{2}\right)$,

using Eq.(8) it becomes

$\Delta E_{\mathrm{Ph}}^{(1)}=i \int_{-a / 2}^{a / 2} d^{3} \mathbf{x} D_{B}(x, x)\left[-i\left(g^{\mu \nu} k^{2}-k^{\mu} k^{v}\right) \delta_{3}\right]$,

where $D_{B}\left(x, x^{\prime}\right)$ is the propagator of electromagnetic field in the bounded space. For overall consistency, we use dimensional regularization to control ultraviolet divergences, and a photon mass $\mu$ to control infrared divergences. Using analogies between an electromagnetic field and a massless scalar field, photon propagator is considered as

$$
\begin{aligned}
& D_{B}\left(x, x^{\prime}\right)=\frac{-2 i g_{\mu \nu}}{a} \int \frac{\mathrm{d} \omega}{2 \pi} \int \frac{d^{d-2} k_{\perp}}{(2 \pi)^{d-2}} \\
& \times \sum_{n} \frac{e^{-i \omega\left(t-t^{\prime}\right)} e^{-i k_{\perp} \cdot\left(x_{\perp}-x_{\perp}^{\prime}\right)} \sin \left(k_{n}\left(z+\frac{a}{2}\right)\right) \sin \left(k_{n}\left(z^{\prime}+\frac{a}{2}\right)\right)}{\omega^{2}-k_{\perp}^{2}-k_{n}^{2}+\mu^{2}} .
\end{aligned}
$$

Here $k_{\perp}$ and $k_{n}$ denote the parallel and the perpendicular momenta to plates (in $z$-direction), respectively. Note that, both contributions related to TE mode and TM mode are considered to be the same, hence the final energy should become twice. After the usual Wick rotation and using Eqs. (19) and (25), with $x=x^{\prime}$, and carrying out the integration over the space then over solid angle in the $d$-dimensional Euclidean space we have

$$
\Delta E_{\mathrm{Ph}}^{(1)}=\frac{12 S \delta_{3} \pi^{\frac{d-1}{2}}}{(2 \pi)^{d-1} \Gamma\left(\frac{d-1}{2}\right)} \int d k_{E} k_{E}^{d-2} \sum_{n} \frac{k_{E}^{2}+k_{n}^{2}}{k_{E}^{2}+k_{n}^{2}+\mu^{2}},
$$

where $S$ is the area of the planes, $k_{E}^{2}=\omega^{2}+k_{\perp}^{2}$ and $k_{n}$ is obtained using the Dirichlet boundary condition on the walls,

$k_{n}=\frac{n \pi}{a}, \quad n=1,2,3, \ldots$
The one-loop photon correction to the vacuum energy in free space is

$\Delta E_{\mathrm{Ph}}^{\prime(1)}=i \int d^{3} \mathbf{x} D_{F}(x, x)\left[-i\left(g^{\mu v} k^{2}-k^{\mu} k^{v}\right) \delta_{3}\right]$,

where $D_{F}\left(x, x^{\prime}\right)$ is the propagator of electromagnetic field in free space in Feynman gauge $(\xi=1)$. We can use a trivial periodic boundary condition on the walls located at $-L / 2$ and $+L / 2$. Carrying out the space integrations gives

$\Delta E_{\mathrm{Ph}}^{\prime(1)}=\frac{12 S L \delta_{3} \pi^{\frac{d-1}{2}}}{(2 \pi)^{d} \Gamma\left(\frac{d-1}{2}\right)} \int d k_{E} k_{E}^{d-2} \int d k \frac{k_{E}^{2}+k^{2}}{k_{E}^{2}+k^{2}+\mu^{2}}$.

To get a vacuum energy comparable with the volume between plates, we should multiply the above energy by a factor $\frac{a}{L}$ then take the limit $L \rightarrow \infty$. We carry out $k_{E}$ integration and import $\delta_{3}$ from Eq. (19). Here we have two types of regulators, $d$ and $\mu$, to control the ultraviolet and infrared divergences, respectively. We first work with $d$ to eliminate some of divergences and derive a result for a photon with mass $\mu$, finally we will approach $\mu$ to zero. As $d \rightarrow 4$ we can cancel the divergent terms using our full freedom to choose two different dimensional regulators $d$ corresponding to free and bounded cases. Then we can perform the integration of $x$ parameter of $\delta_{3}$ to get

$$
\begin{aligned}
& \Delta E_{\mathrm{Cas} .}^{\mathrm{Ph}}=\Delta E_{\mathrm{Ph}}^{(1)}-\Delta E_{\mathrm{Ph}}^{\prime(1)}+\mathcal{O}\left(\alpha^{2}\right)=\frac{2 \alpha S \mu^{\prime 2}}{a^{3}} \\
& \quad \times\left[\sum_{n=1} \sqrt{n^{2}+\mu^{\prime 2}}\left(\gamma-1+\ln \sqrt{n^{2}+\mu^{\prime 2}}\right)\right. \\
& \left.\quad-\int_{0}^{\infty} d k^{\prime} \sqrt{k^{\prime 2}+\mu^{\prime 2}}\left(\gamma-1+\ln \sqrt{k^{\prime 2}+\mu^{\prime 2}}\right)\right]+\mathcal{O}\left(\alpha^{2}\right),
\end{aligned}
$$

where $\gamma$ is the Euler-Mascheroni number and we have changed the variables as $k^{\prime}=\frac{a k}{2 \pi}$ and $\mu^{\prime}=\frac{a \mu}{2 \pi}$. Now, we can use the Abel-Plana summation formula (APSF) [56], which basically converts our summation into an integration,

$$
\begin{aligned}
& \sum_{n=1}^{\infty} f(n)=-\frac{f(0)}{2}+\int_{0}^{\infty} f(x) \mathrm{d} x \\
& +i \int_{0}^{\infty} \frac{\mathrm{d} t}{e^{2 \pi t}-1}[f(i t)-f(-i t)] .
\end{aligned}
$$

Apply this formula for Eq. (30) yields (see Appendix for details)

$$
\begin{aligned}
& \Delta E_{\text {Cas. }}^{\mathrm{Ph}}=\frac{2 S \alpha \mu^{\prime 2}}{a^{3}}\left[-\frac{\mu^{\prime}}{2}\left(\gamma-1+\ln \mu^{\prime 2}\right)\right. \\
& \left.\quad+2 \int_{\mu^{\prime}}^{\infty} \frac{\mathrm{d} t}{e^{2 \pi t}-1} \sqrt{t^{2}-\mu^{\prime 2}}\left(\gamma-1+\ln \sqrt{t^{2}-\mu^{\prime 2}}\right)\right] \\
& \quad+\mathcal{O}\left(\alpha^{2}\right) .
\end{aligned}
$$


It is obvious that as $\mu$ tends to zero, $E_{\mathrm{Cas}}^{\mathrm{Ph}}$ approaches zero, up to order $\alpha$ :

$\lim _{\mu \rightarrow 0} \Delta E_{\text {Cas. }}^{\mathrm{Ph}}=0$.

Therefore, the photonic loop does not contribute to $\mathcal{O}(\alpha)$ radiative correction to the Casimir energy.

\section{Fermionic Loop}

In this section, we calculate NLO radiative correction to the Casimir energy due to fermionic loop $\otimes$. We use the MIT bag boundary condition on the plates. According to MIT bag boundary condition there is no flux of fermions through the boundary, this means that

$n_{\mu} j^{\mu}=0$,

where $j^{\mu}$ indicates the current of the Dirac field and $n_{\mu}$ is the normal unit vector to the boundary, or more strictly it implies to complete confinement of the spinor field. Note that, ideal conductor boundary condition for the electromagnetic field and bag boundary conditions for the spinor field can go together. This can be seen from the field equations (Maxwell equations) written in the form

$\partial_{\mu} F^{\mu \nu}=e \bar{\psi} \gamma^{v} \psi$

after multiplying with the normal vector $n_{v}$

$\partial_{\mu} n_{\nu} F^{\mu \nu}=e \bar{\psi} n_{\nu} \gamma^{\nu} \psi$

Dirichlet boundary condition on the walls vanishes the left side, so that we can use the bag boundary condition. Then MIT bag boundary condition turns out to be [58-61]

$[1+i(\hat{\mathbf{n}} \cdot \boldsymbol{\gamma})] \psi(\mathbf{x})=0$,

which is satisfied on the boundary, more accurate on the plates. Applying this condition to Dirac spinor field, one can derive

$p a \cot (p a)=-m a$,

which determines quantized modes. Two limits are interesting to calculate; small mass and large mass limits. As a matter of fact, the mass is small (large) in comparison with the distance $a$, i.e. $m a \ll 1(m a \gg 1)$. For small mass limit the solutions of Eq. (39) are (see for more details [62])

$p_{n}=\left(n+\frac{1}{2}\right) \frac{\pi}{a} \quad$ with $\quad n=0,1,2, \ldots$ where $p_{n}$ denotes the parallel momenta to the plates (in $z$ direction). Now, again for the bounded space we have

$$
\begin{aligned}
\Delta E_{\mathrm{F}} & =\int_{-a / 2}^{a / 2} d^{3} x\left\langle\Omega\left|\mathcal{H}_{I}\right| \Omega\right\rangle=i \int_{-a / 2}^{a / 2} \bigotimes d^{3} x+\mathcal{O}\left(\alpha^{2}\right) \\
& =i \int_{-a / 2}^{a / 2} d^{3} x \operatorname{Tr}\left[S_{B}(x, x) i\left(\not \varnothing \delta_{2}-\delta_{m}\right)\right]+\mathcal{O}\left(\alpha^{2}\right),
\end{aligned}
$$

where $S_{B}\left(x, x^{\prime}\right)$, the Feynman propagator of spinor field between plates, is

$$
\begin{aligned}
& S_{B}\left(x, x^{\prime}\right)=\frac{i}{a} \int \frac{\mathrm{d} \omega}{2 \pi} \int \frac{d^{2} p_{\perp}}{(2 \pi)^{2}} \\
& \times \sum_{n=0} \frac{\not p+m}{\omega^{2}-p_{\perp}^{2}-p_{n}^{2}-m^{2}+i \epsilon} \\
& \times e^{-i \omega\left(t-t^{\prime}\right)} e^{-i p_{\perp}\left(x_{\perp}-x^{\prime}\right)} e^{-i p_{n}\left(z-z^{\prime}\right)} .
\end{aligned}
$$

Here $p_{\perp}$ and $p_{n}$ indicate the parallel and the perpendicular momenta to the plates, respectively. Converting the integrals into dimensionless form in $d$ spacetime dimensions we have

$$
\begin{aligned}
S_{B}\left(x, x^{\prime}\right)= & \frac{i}{a^{d-1}} \int \frac{\mathrm{d} \tilde{\omega}}{2 \pi} \int \frac{d^{d-2} \tilde{p}_{\perp}}{(2 \pi)^{d-2}} \\
& \times \sum_{n=0} \frac{\tilde{p}+\tilde{m}}{\tilde{\omega}^{2}-\tilde{p}_{\perp}^{2}-p_{n}^{2}-\tilde{m}^{2}+i \tilde{\epsilon}} e^{-i \frac{\tilde{\omega}}{a}\left(t-t^{\prime}\right)} \\
& \times e^{-i \frac{\tilde{p}_{\perp}}{a}\left(x_{\perp}-x_{\perp}^{\prime}\right)} e^{-i \frac{\tilde{p}_{n}}{a}\left(z-z^{\prime}\right)}
\end{aligned}
$$

After the usual Wick rotation and carrying out the integration, one can obtain

$$
\begin{aligned}
& \Delta E_{\mathrm{F}}^{(1)}=\frac{32 S}{(2 \pi)^{d-1} a^{d-1}} \frac{\pi^{\frac{d-1}{2}}}{\Gamma\left(\frac{d-1}{2}\right)} \int \mathrm{d} \tilde{p}_{E} \tilde{p}_{E}^{d-2} \\
& \quad \times \sum_{n=0}\left(\frac{\tilde{p}_{E}^{2}+\tilde{p}_{n}^{2}}{\tilde{p}_{E}^{2}+\tilde{p}_{n}^{2}+\tilde{m}^{2}} \delta_{2}-\frac{\tilde{m}}{\tilde{p}_{E}^{2}+\tilde{p}_{\perp}^{2}+\tilde{p}_{n}^{2}+\tilde{m}^{2}} \delta m\right),
\end{aligned}
$$

where $\tilde{p}_{E}^{2}=\tilde{\omega}^{2}+\tilde{p}_{\perp}^{2}$. Similarly, for the free space we have

$$
\Delta E_{\mathrm{F}}^{\prime(1)}=i \int d^{3} x \operatorname{Tr}\left[S_{F}(x, x) i\left(\not p \delta_{2}-\delta_{m}\right)\right]
$$

Using Eq. (9) for the free propagator and after integration we obtain

$$
\begin{aligned}
& \Delta E_{\mathrm{F}}^{\prime(1)}=\frac{32 S}{(2 \pi)^{d-1} a^{d-2}} \frac{\pi^{\frac{d-1}{2}}}{\Gamma\left(\frac{d-1}{2}\right)} \int \mathrm{d} \tilde{p}_{E} \tilde{p}_{E}^{d-2} \\
& \quad \times \int \frac{\mathrm{d} \tilde{p}}{2 \pi}\left(\frac{\tilde{p}_{E}^{2}+\tilde{p}^{2}}{\tilde{p}_{E}^{2}+\tilde{p}^{2}+\tilde{m}^{2}} \delta_{2}-\frac{\tilde{m}}{\tilde{p}_{E}^{2}+\tilde{p}^{2}+\tilde{m}^{2}} \delta_{m}\right) .
\end{aligned}
$$


For the small mass case the radiative correction to Casimir energy corresponding to the fermionic loop becomes

$$
\begin{aligned}
\Delta E_{\text {Cas. }}^{\mathrm{F}}= & \Delta E_{\mathrm{F}}^{(1)}-\Delta E_{\mathrm{F}}^{\prime(1)}+\mathcal{O}\left(\alpha^{2}\right) \\
= & \frac{32 S \pi^{\frac{d-1}{2}}}{(2 \pi)^{d-1} \Gamma\left(\frac{d-1}{2}\right)} \frac{\pi^{d-1}}{a^{d-1}} \int \mathrm{d} p_{E}^{\prime} p_{E}^{\prime d-2} \\
& \times\left[\delta _ { 2 } \left(\sum_{n=0} \frac{\left(n+\frac{1}{2}\right)^{2}+p_{E}^{\prime 2}}{\left(n+\frac{1}{2}\right)^{2}+p_{E}^{\prime 2}+m^{\prime}}\right.\right. \\
& \left.-\frac{1}{2} \int \mathrm{d} p^{\prime} \frac{p^{\prime 2}+p_{E}^{\prime 2}}{p^{\prime 2}+p_{E}^{\prime 2}+m^{\prime 2}}\right) \\
& -\frac{a m^{\prime} \delta_{m}}{\pi}\left(\sum_{n=0} \frac{1}{\left(n+\frac{1}{2}\right)^{2}+p_{E}^{\prime 2}+m^{\prime 2}}\right. \\
& \left.\left.-\frac{1}{2} \int \mathrm{d} p^{\prime} \frac{1}{p^{\prime 2}+p_{E}^{\prime 2}+m^{\prime 2}}\right)\right]+\mathcal{O}\left(\alpha^{2}\right),
\end{aligned}
$$

where we use the change of variables as $p^{\prime}=\tilde{p} / \pi, p_{E}^{\prime}=$ $\tilde{p}_{E} / \pi$ and $m^{\prime}=\tilde{m} / \pi$. Integrating of $p_{E}^{\prime}$ yields

$$
\begin{aligned}
& \Delta E_{\text {Cas. }}^{\mathrm{F}}=\frac{32 S \pi^{\frac{d-1}{2}}}{2^{d-1} a^{d-1} \Gamma\left(\frac{d-1}{2}\right)} \frac{1}{2} \sec \left(\frac{\mathrm{d} \pi}{2}\right) \\
& \quad \times \pi\left\{\delta _ { 2 } m ^ { \prime 2 } \left[\sum_{n=0}^{\infty}\left[(n+1 / 2)^{2}+m^{\prime 2}\right]^{\frac{d-3}{2}}\right.\right. \\
& \left.-\int_{0}^{\infty}\left[p^{\prime 2}+m^{\prime 2}\right]^{\frac{d-3}{2}} \mathrm{~d} p^{\prime}\right] \\
& +\frac{a m^{\prime} \delta_{m}}{\pi}\left[\sum_{n=0}^{\infty}\left[(n+1 / 2)^{2}+m^{\prime 2}\right]^{\frac{d-3}{2}}\right. \\
& \left.\left.-\int_{0}^{\infty}\left[p^{\prime 2}+m^{\prime 2}\right]^{\frac{d-3}{2}} \mathrm{~d} p^{\prime}\right]\right\}+\mathcal{O}\left(\alpha^{2}\right) .
\end{aligned}
$$

Here we need another type of APSF to convert the sum into integral,

$$
\begin{aligned}
& \sum_{n=0}^{\infty} f\left(n+\frac{1}{2}\right)=\int_{0}^{\infty} f(x) \mathrm{d} x \\
& \quad-i \int_{0}^{\infty} \frac{\mathrm{d} t}{e^{2 \pi t}+1}[f(i t)-f(-i t)] .
\end{aligned}
$$

We can use the following formula to calculate the branch cut integral: if $\left.f(z)=\left(z^{n}+\alpha^{m}\right)\right)^{p / 2}$

$$
\begin{aligned}
& i \int_{0}^{\infty} \frac{f(i t)-f(-i t)}{e^{2 \pi t}+1} d t \\
& \quad=-2 \sin \left(\frac{p n \pi}{4}\right) \int_{\alpha^{m / n}}^{\infty} \frac{\left(t^{n}-\alpha^{m}\right)^{p / 2}}{e^{2 \pi t}+1} d t .
\end{aligned}
$$

In addition we know that

$$
\frac{1}{e^{2 \pi t}+1}=\sum_{j=1}^{\infty}(-1)^{j+1} e^{-2 \pi j t} \text {. }
$$

We use these formulae, and import $\delta_{m}$ and $\delta_{2}$ from Eqs. (20) and (21), respectively, into Eq. (48). Again, similar to the procedure adopted in photonic loop, which lad to Eq. (30), we first expand the expression about $d=4$ then take the limit $d \rightarrow 4$. No divergent term remains due to the usual subtraction in Casimir effect. We then, do the $x$ integration. Finally, taking the limit $\mu \rightarrow 0$ we obtain

$$
\begin{aligned}
& \Delta E_{\text {Cas. }}^{\mathrm{F}+\mathrm{Ph}}=\Delta E_{\text {Cas. }}^{\mathrm{F}}+\Delta E_{\text {Cas. }}^{\mathrm{Ph}}=\sum_{j=1}^{\infty}-\frac{(-1)^{j} 5 S \alpha m^{2}}{16 j^{2} \pi^{4} a} \\
& \times\left\{K_{0}(2 j a m)+2 j_{a m} K_{1}(2 j a m)\left[\frac{14}{5} \ln (m a)+N_{j}\right]\right\},
\end{aligned}
$$

where $N_{j}=\gamma-\ln \left(j \pi^{2}\right)-\frac{12+\ln 8}{5}$. This is the final result of the radiative correction to the Casimir energy due to fermionic loop, for the small mass case.

The other interesting limit is the large mass limit. In this case, Eq. (39) turns out to be

$m a \tan (p a)=-p a$.

Now, the solutions are

$p_{n}=\frac{n \pi}{a}$ with $n=1,2, \ldots$

We follow the similar way for obtaining Eq. (48), but now we should apply APSF Eq. (31) and need the following relation

$\frac{1}{e^{2 \pi t}-1}=\sum_{j=1}^{\infty} e^{-2 \pi j t}$.

Finally, our the radiative NLO correction to Casimir energy in this case becomes

$$
\begin{aligned}
& \Delta E_{\text {Cas. }}^{\mathrm{F}+\mathrm{Ph}}=\Delta E_{\text {Cas. }}^{\mathrm{F}}+\Delta E_{\text {Cas. }}^{\mathrm{Ph}}=\sum_{j=1}^{\infty} \frac{5 S \alpha m^{2}}{16 j^{2} \pi^{4} a} \\
& \quad \times\left\{K_{0}(2 j a m)+2 j_{a m K_{1}}(2 j a m)\left[\frac{14}{5} \ln (\mathrm{ma})+N_{j}\right]\right\}
\end{aligned}
$$

The first term, in Eq. (31) (i.e. $+f(0) / 2)$ turns out to be independent of distance between plates $a$. Therefore this term has no impact on the physics of problem and we ignore it. For the large mass case which is also equivalent to the large distances, Eq. (56) takes the form

$\Delta E_{\text {Cas. }}^{\mathrm{F}+\mathrm{Ph}} \sim \frac{23 S \alpha}{16} \pi^{-7 / 2} m^{5 / 2} a^{-1 / 2} \ln ($ am $) e^{-2 a m}$.

The pressure on the plates related to this term is

$\Delta P_{\text {Cas. }}^{\mathrm{F}+\mathrm{Ph}} \sim \frac{23 \alpha}{8} \pi^{-7 / 2} m^{3} \sqrt{m / a} \ln (a m) e^{-2 a m}$,

which clearly shows the exponentially damping structure. In Figs. 1 and 2 we compare our result with the leading terms of the Casimir energy for electromagnetic and fermion fields, 


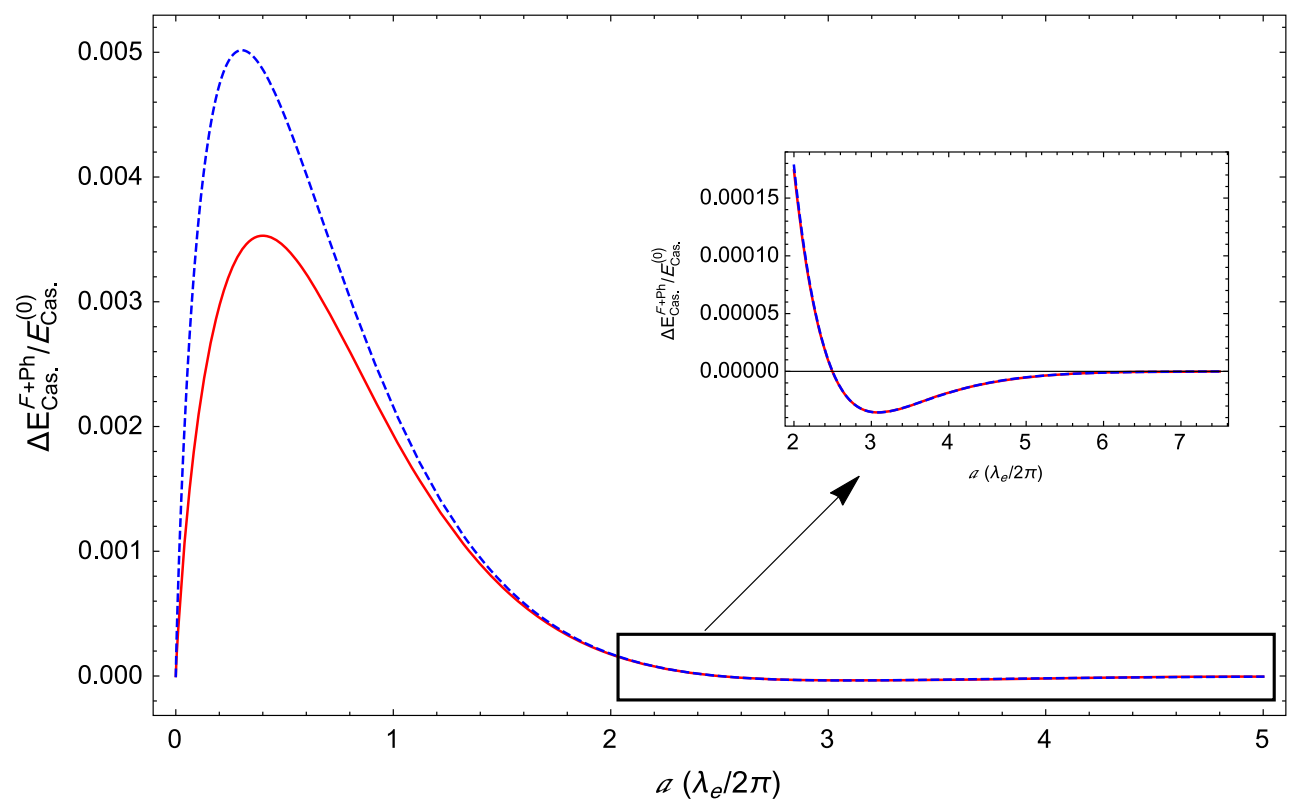

Fig. 1 The ratio between the one-loop correction derived here and the leading term of electromagnetic Casimir energy $E_{\text {Cas. }}^{\mathrm{F}+\mathrm{Ph}} / E_{\mathrm{Cas} .}^{(0)}$, vs the plates separation ( $\lambda_{e}$ denotes the Compton wavelength of electron.) Solid (dashed) line shows the large (small) mass limit

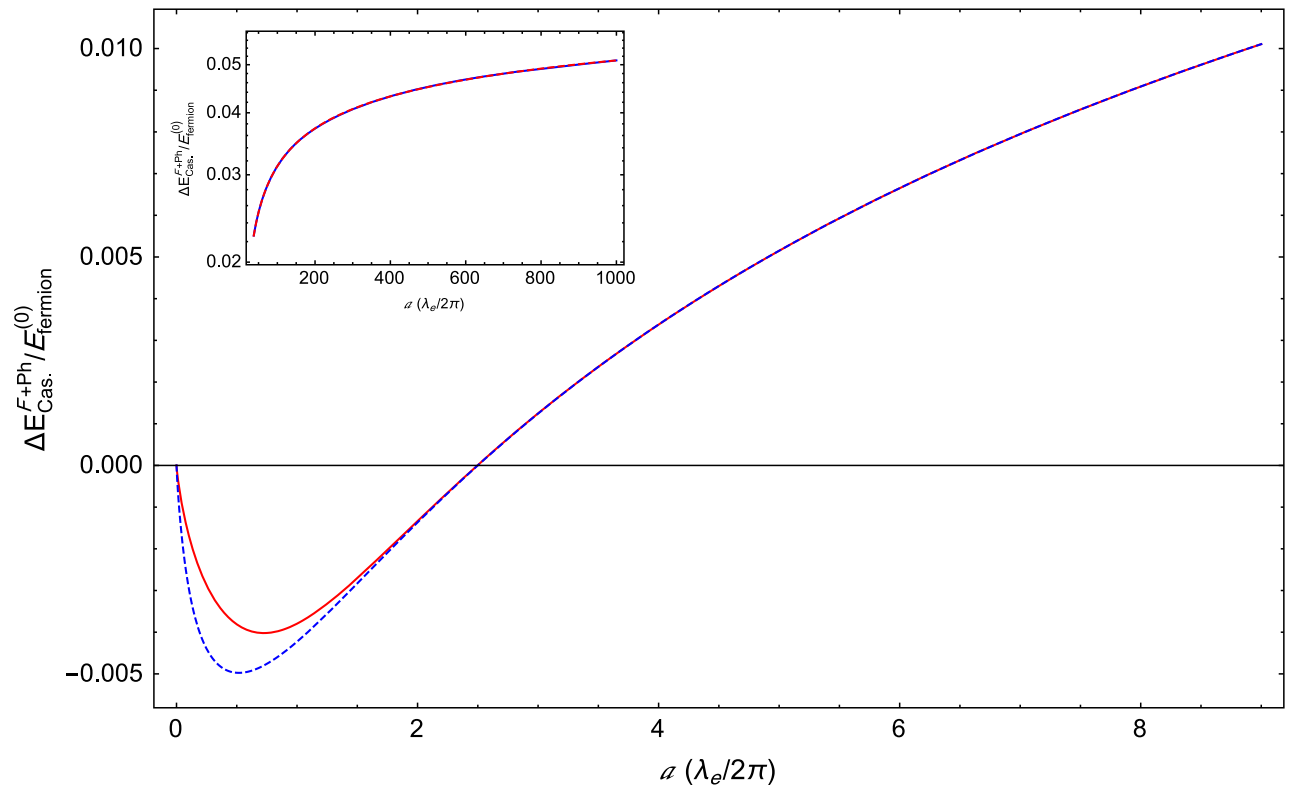

Fig. 2 The ratio between the one-loop correction derived here and the leading term of fermionic Casimir energy $E_{\mathrm{Cas}}^{\mathrm{F}+\mathrm{Ph}} / E_{\mathrm{Fermion}}^{(0)}$, vs the plates separation ( $\lambda_{e}$ denotes the Compton wavelength of electron.) Solid (dashed) line shows the large (small) mass limit

respectively. Figure 1 shows that the computed correction is negligible even in very small separations. In Fig. 2 we see that the impact of this correction increases in large separations.

\section{Conclusions}

We have calculated one-loop radiative correction to the Casimir energy due to photonic and fermionic counterterms within the renormalized perturbation theory for QED theory. The topology considered here is two perfectly conducting parallel plates in $(3+1)$ dimensions. We have used Dirichlet boundary condition for Electromagnetic field and MIT bag boundary condition for electron. To control ultraviolet divergences we have used dimensional regularization and a photon mass $\mu$ also is used to control infrared divergences. It is found that photonic loop does not have any contribution up to order $\alpha$. The force per unit area related to fermionic and photonic 
loops, up to this order, at large distances have been obtained as $\Delta P_{\mathrm{F}}^{\text {Cas. }} \sim-\frac{23 \alpha}{8} \pi^{-7 / 2} m^{3} \sqrt{m a} \ln (a m) e^{-2 a m}$. We illustrate our result in Figs. 1 and 2 and compare it with the related leading Casimir energy of electromagnetic and fermion field.

Acknowledgments It is a great pleasure for us to acknowledge the useful discussion and comments of S.M. Fazeli and M.M. Ettefaghi. This research was supported by the office of research of the University of Qom.

Open Access This article is distributed under the terms of the Creative Commons Attribution 4.0 International License (http://creativecomm ons.org/licenses/by/4.0/), which permits unrestricted use, distribution, and reproduction in any medium, provided you give appropriate credit to the original author(s) and the source, provide a link to the Creative Commons license, and indicate if changes were made.

Funded by SCOAP ${ }^{3}$.

\section{Appendix: Calculation of the branch-cut terms}

In this Appendix we calculate two types of branch-cut terms which appear in Eq. (30). Regardless of some constants, this equation is of the form

$$
\begin{aligned}
& \sum_{n=1} \sqrt{n^{2}+b^{2}}\left(C+\ln \sqrt{n^{2}+b^{2}}\right) \\
& -\int_{0}^{\infty} \mathrm{d} x \sqrt{x^{2}+b^{2}}\left(C+\ln \sqrt{x^{2}+b^{2}}\right)
\end{aligned}
$$

In the APSF

$$
\begin{aligned}
\sum_{n=1}^{\infty} f(n)= & -\frac{f(0)}{2}+\int_{0}^{\infty} f(x) \mathrm{d} x \\
& +i \int_{0}^{\infty} \frac{\mathrm{d} t}{e^{2 \pi t}-1}[f(i t)-f(-i t)],
\end{aligned}
$$

Assuming $f(x)=\sqrt{x^{2}+b^{2}}\left(C+\ln \sqrt{x^{2}+b^{2}}\right)$ we can write

$$
\begin{aligned}
f(i t)-f(-i t)= & C\left(\sqrt{b^{2}+(i t)^{2}}-\sqrt{b^{2}+(-i t)^{2}}\right) \\
& +\left(\sqrt{b^{2}+(i t)^{2}} \ln \sqrt{b^{2}+(i t)^{2}}\right. \\
& \left.-\sqrt{b^{2}+(-i t)^{2}} \ln \sqrt{b^{2}+(-i t)^{2}}\right),
\end{aligned}
$$

Choosing, $b=|b| e^{i \theta_{b}}, t=|t| e^{i \theta_{t}}$, we have for the first term

$$
\begin{aligned}
\sqrt{b^{2}+(i t)^{2}}-\sqrt{b^{2}+(-i t)^{2}}= & \sqrt{|b| e^{i 2 \theta_{b}}+e^{i \pi}|t|^{2} e^{i 2 \theta_{t}}} \\
& -\sqrt{|b| e^{i 2 \theta_{b}}+e^{-i \pi}|t|^{2} e^{i 2 \theta_{t}}} \\
= & \left(e^{\frac{i \pi}{2}} e^{i \theta_{t}}-e^{\frac{-i \pi}{2}} e^{i \theta_{t}}\right) \\
& \times \sqrt{|b| e^{i\left(2 \theta_{b}+\pi-2 \theta_{t}\right)}+|t|^{2}} \\
= & 2 i \sin \left(\frac{\pi}{2}\right) \sqrt{t^{2}-b^{2}} .
\end{aligned}
$$

where one should note that $e^{i\left(2 \theta_{b}+\pi-2 \theta_{t}\right)}=-1$ and we assume $t>|b|$. For $t<|b|$ this term is exactly zero. Similarly for second term, we have

$$
\begin{aligned}
& \sqrt{b^{2}+(i t)^{2}} \ln \sqrt{b^{2}+(i t)^{2}} \\
& -\sqrt{b^{2}+(-i t)^{2}} \ln \sqrt{b^{2}+(-i t)^{2}} \\
& =\sqrt{b^{2}+e^{i \pi} t^{2}} \ln \sqrt{b^{2}+e^{i \pi} t^{2}} \\
& -\sqrt{b^{2}+e^{-i \pi} t^{2}} \ln \sqrt{b^{2}+e^{-i \pi} t^{2}} \\
& \left.=\sqrt{b^{2}+e^{i \pi} t^{2}} \ln \left(e^{i \pi / 2} \sqrt{e^{-i \pi} b^{2}+t^{2}}\right)\right) \\
& -\sqrt{b^{2}+e^{-i \pi} t^{2}} \ln \left(e^{-i \pi / 2} \sqrt{b e^{i \pi}+t^{2}}\right) \\
& =i \frac{\pi}{2}\left[\sqrt{b^{2}+e^{i \pi} t^{2}}+\sqrt{b^{2}+e^{-i \pi} t^{2}}\right] \\
& +\left[\sqrt{b^{2}+e^{i \pi} t^{2}}-\sqrt{b^{2}+e^{-i \pi} t^{2}}\right] \ln \sqrt{t^{2}-b^{2}} \text {. }
\end{aligned}
$$

Now, the first of the last line is similar to similar to (61) but with plus sign between its terms, so we get

$$
\sqrt{b^{2}+e^{i \pi} t^{2}}+\sqrt{b^{2}+e^{-i \pi} t^{2}}=2 \sqrt{t^{2}-b^{2}} \cos \frac{\pi}{2}=0 .
$$

For the second term, using Eqs. (61) and (63), we have for $t<|b|$

$$
\begin{aligned}
& \sqrt{b^{2}+e^{i \pi} t^{2}} \ln \left(b^{2}+e^{i \pi} t^{2}\right)-\sqrt{b^{2}+e^{-i \pi} t^{2}} \\
& \quad \times \ln \left(b^{2}+e^{-i \pi} t^{2}\right)=2 i \sqrt{t^{2}-b} \ln \sqrt{t^{2}-b^{2}} .
\end{aligned}
$$

For $t<|b|$ this term is exactly zero. Therefore, our final result derived as follows:

$$
\begin{aligned}
& i \int_{0}^{\infty} \frac{\mathrm{d} t}{e^{2 \pi t}-1}[f(i t)-f(-i t)] \\
& \quad=2 \int_{b}^{\infty} \frac{\mathrm{d} t}{e^{2 \pi t}-1} \sqrt{t^{2}-b^{2}}\left(C-\ln \sqrt{t^{2}-b^{2}}\right) .
\end{aligned}
$$

\section{References}

1. H.B.G. Casimir, D. Polder, The influence of retardation on the London-van der Waals forces. Phys. Rev. 73, 360 (1948)

2. M.J. Sparnaay, Measurements of attractive forces between flat plates. Physica 24, 751 (1958)

3. W. Arnopld, S. Hunklinger, K. Dransfeld, Phys. Rev. B 19, 6049 (1979)

4. M. Bordag, U. Mohideen, V.M. Mostepanenko, New developments in the Casimir effect. Phys. Rep. 353(1-3), 1 (2001). arXiv:quant-ph/0106045

5. K. A. Milton, The Casimir effect: physical manifestations of zeropoint energy (World Scientific Publishing Co., 2001)

6. S.K. Lamoreaux, Demonstration of the Casimir force in the 0.6 to 6 m range. Phys. Rev. Lett. 78, 5 (1997)

7. G. Bressi, G. Carugno, R. Onfrio, G. Ruoso, Measurement of the Casimir force between parallel metallic surfaces. Phys. Rev. Lett. 88, 041804 (2002)

8. A. Gusso, A.G.M. Schmidt, Repulsive Casimir forces produced in rectangular cavities: possible measurements and applications. Braz. J. Phys. 36(1B), 168 (2006) 
9. D. Garcia-Sanchez, K.Y. Fong, H. Bhaskaran, S. Lamoreaux, H.X. Tang, Casimir force and in situ surface potential measurements on nanomembranes. Phys. Rev. Lett. 109, 027202 (2012)

10. W.J. Kim, A.O. Sushkov, D.A.R. Dalvit, S.K. Lamoreaux, Surface contact potential patches and Casimir force measurements. Phys. Rev. A 81, 022505 (2010)

11. S.K. Lamoreaux, Reanalysis of Casimir force measurements in the 0.6-to-6-m range. Phys. Rev. A 82, 024102 (2010)

12. E. Elizalde, Matching the observational value of the cosmological costant. Phys. Lett. B 516, 143 (2001)

13. F. Bauer, M. Lindner, G. Seidl, Casimir energy in deconstruction and the cosmological constant. JHEP 05, 026 (2004)

14. G. Mahajan, S. Sarkar, T. Padmanabhan, Casimir effect confronts cosmologica lconstant. Phys. Lett. B 641 (2006)

15. M. Fabinger, P. Horava, Casimir effect between world-branes in heterotic M-theory. Nucl. Phys. B 580, 243 (2000)

16. K. Poppenhaeger, S. Hossenfelder, S. Hofmann, M. Bleicher, The Casimir effect in the presence of compactified universal extra dimensions. Phys. Lett. B 582, 1 (2004)

17. L.P. Teo, Casimir effect in spacetime with extradimensions from Kaluza-Klein to Randall-Sundrummodels. Phys. Lett. B 682 (2009)

18. R. Linares, H.A. Morales-Tecotl, O. Pedraza, Casimir force in brane worlds: coinciding results from Green's and zeta function approaches. Phys. Rev. D 81, 126013 (2010)

19. N.A. Kawakami, M.C. Nemes, F. Walter, Wreszinski, The Casimir effect for parallel plates revisited. J. Math. Phys. 48, 102302 (2007)

20. S. Hacyan, R. Jauregui, C. Villarreal, Spectrum of quantum electromagnetic fluctuations in rectangular cavities. Phys. Rev. A 47, 4204 (1993)

21. H. Cheng, The Casimir energy for a rectangular cavity at finite temperature. J. Phys. A Math. Gen. 35, 2205 (2002)

22. X. Li, X. Zhai, Rigorous proof of the attractive nature for the Casimir force of a p-odd hypercube. J. Phys. A Math. Gen. 34, 11053 (2001)

23. G.J. Maclay, Analysis of zero-point electromagnetic energy and Casimir forces in conducting rectangular cavities. Phys. Rev. A 61, 052110 (2000)

24. W. Lukosz, Electromagnetic zero-point energy and radiation pressure for a rectangular cavity. Physica 56, 109 (1971)

25. J.R. Ruggiero, A. Villani, A.H. Zimerman, Some comments on the application of analytic regularisation to the Casimir forces. J. Phys. A Math. Gen. 13, 761 (1980)

26. H. Ahmedov, I.H. Duru, New Casimir energy calculations for single cavities. In Non-linear dynamics and fundamental interactions. NATO Science Series II: Mathematics, Physics and Chemistry, vol. 213 (Springer, Netherlands, 2006), pp. 269-275

27. M. Bordag, G.L. Klimchitskaya, U.Mohideen, V.M. Mostepanenko, Advances in the Casimir effect (Oxford University press, 2009)

28. M.A. Valuyan, R. Moazzemi, S.S. Gousheh, A direct approach to the electromagnetic Casimir energy in a rectangular waveguide. J. Phys. B 41, 145502 (2008)

29. P.A.M. Neto, The dynamical Casimir effect with cylindrical waveguides. J. Opt. B Quantum Semiclass. 7, s86 (2005)

30. F.D. Mazzitelli, M.J. Sanchez, N.N. Scoccola, J. von Stecher, Casimir interaction between two concentric cylinders: exact versus semiclassical results. Phys. Rev. A 67, 013807 (2003)

31. D.A.R. Dalvit, F.C. Lombardo, F.D. Mazzitelli, R. Onofrio, Exact Casimir interaction between concentric cylinders. Phys. Rev. A 74, 020101 (2006)

32. M. Bordag, E. Elizalde, K. Kirsten, S. Leseduarte, Casimir energies for massive scalar fields in a spherical geometry. Phys. Rev. D 56, 4896 (1997)

33. C.M. Bender, K.A. Milton, Scalar Casimir effect for a Ddimensional sphere. Phys. Rev. D 50, 6547 (1994)
34. M. Özcan, Scalar Casimir effect between two concentric spheres. Int. J. Mod. Phys. A 27, 1250082 (2012)

35. M. Bordag, D. Robaschik, E. Wieczorek, Ann. Phys. 165, 192 (1985)

36. M. Bordag, J. Lindig, Radiative correction to the Casimir force on a sphere. Phys. Rev. D 58, 045003 (1998). arXiv:hep-th/9801129

37. D. Robaschik, K. Scharnhorst, E. Wieczorek, Radiative corrections to the Casimir pressure under he influence of temperature and external fields. Ann. Phys. (NY) 174, 401 (1987)

38. M. Bordag, K. Scharnhorst, $\mathcal{O}(\alpha)$ Radiative correction to the Casimir energy for penetrable mirrors. Phys. Rev. Lett. 81, 3815 (1998). arXiv:hep-th/9807121

39. S. S. Xue, Casimir effect of scalar field on $S(n-1)$ manifold. Commun. Theor. Phys. (Wuhan) 11, 243 (1989)

40. F. Ravndal, J.B. Thomassen, Radiative corrections to the Casimir energy and effective field theory. Phys. Rev. D 63, 113007 (2001)

41. X. Kong, F. Ravndal, Radiative corrections to the Casimir energy. Phys. Rev. Lett. 79, 545 (1997)

42. K. Melnikov, Radiative corrections to the Casimir force and effective field theories. Phys. Rev. D 64, 045002 (2001)

43. R.M. Cavalcanti, C. Farina, F.A. Barone, Radiative corrections to Casimir effect in the $\lambda \phi^{4}$ model. (2006). arXiv:hep-th/0604200

44. F.A. Barone, R.M. Cavalcanti, C. Farina, Radiative corrections to the Casimir effect for the massive scalar field. (2003). arXiv:hep-th/0301238v1

45. F.A. Barone, R.M. Cavalcanti, C. Farina, Radiative corrections to the Casimir effect for the massive scalar field. Nucl. Phys. Proc. Suppl. 127, 118 (2004). arXiv:hep-th/0306011v2

46. R. Moazzemi, S.S. Gousheh, A new renormalization approach to the Dirichlet Casimir effect for $\phi^{4}$ theory in $(1+1)$ dimensions. Phys. Lett. B, 658 (2008)

47. S.S. Gousheh, R. Moazzemi, M.A. Valuyan, Radiative correction to the Dirichlet Casimir energy for $\lambda \phi^{4}$ theory in two spatial dimensions, Phys. Lett. B, 681 (2009)

48. R. Moazzemi, M. Namdar, S.S. Gousheh, The Dirichlet Casimir effect for $\phi^{4}$ theory in $(3+1)$ dimensions: a new renormalization approach. JHEP 0709, 029 (2007)

49. L.H. Ford, Proc. R. Soc. Lond. A 368, 305 (1979)

50. B.S. Kay, Phys. Rev. D 20, 3052 (1979)

51. D.J. Toms, Phys. Rev. D 21, 2805 (1980)

52. K. Langfeld, F. Schmuser, H. Reinhardt, Phys. Rev. D 51, 765 (1995)

53. L.C. de Albuquerque, Phys. Rev. D 55, 7754 (1997)

54. M.V. Cougo-Pinto, C. Farina, A. Tort, Lett. Math. Phys. 38, 97 (1996)

55. M.E. Peskin, D.V. Schroeder, An introduction to Quantum Field Theory (Addison-Wesley Publishing Company, 1995)

56. P. Henrici, in Applied and computational complex analysis, vol. 1, (Wiley, New York, 1984), ed. by E.T. Whittaker, G.N. Watson, A Course of Modern Analysis, (Cambridge University Press, 1958)

57. A.A. Saharian, arXiv:hep-th/0002239, arXiv:0708.1187

58. P.N. Bogolioubov, Ann. Inst. Henri Poincare 8, 163 (1967)

59. A. Chodos, R.L. Jaffe, K. Johnson, C.B. Thorn, V.F. Weisskopf, Phys. Rev. D 9, 3471 (1974)

60. A. Chodos, R.L. Jaffe, K. Johnson, C.B. Thorn, Phys. Rev. D 10, 2599 (1974)

61. R. Saghian, M.A. Valuyan, A. Seyedzahedi, S.S. Gousheh, Casimir energy for a massive Dirac field in one spatial dimension: a direct approach. Int. J. Mod. Phys. A 27, 1250038 (2012)

62. A. Seyedzahedi, R. Saghian, S.S. Gousheh, Casimir energy for a massless fermionic field confined inside a three-dimensional. Phys. Rev. A 82, 032517 (2010) 